This is a peer-reviewed, accepted author manuscript of the following article: Freerks, L., Papadatou Soulou, E., Batchelor, H., \& Klein, S. (2019). A review of GI conditions critical to oral drug absorption in malnourished children. European Journal of Pharmaceutics and Biopharmaceutics , 137, 9-22. https://doi.org/10.1016/j.ejpb.2019.02.001

\title{
A review of $\mathrm{Gl}$ conditions critical to oral drug absorption in malnourished children
}

Lisa Freerks ${ }^{1}$, Eleni Papadatou Soulou², Hannah Batchelor ${ }^{2}$, Sandra Klein ${ }^{1 *}$

${ }^{1}$ Department of Pharmacy, University of Greifswald, 17489 Greifswald, Germany

${ }^{2}$ School of Pharmacy, College of Medical and Dental Sciences, University of Birmingham, Birmingham B15 2TT, UK

${ }^{*}$ Corresponding author:

Prof. Dr. Sandra Klein

University of Greifswald

Department of Pharmacy

Institute of Biopharmaceutics and Pharmaceutical Technology

Center of Drug Absorption and Transport

3 Felix Hausdorff Street

Greifswald, 17489

Germany

Tel.: ++49-3834-420-4897

Fax: ++49-3834-420-4886

e-mail: Sandra.Klein@uni-greifswald.de 


\section{Abstract}

Accurate prediction of oral absorption of drugs relies on biorelevant methodology. Current methods are based on Western healthy adult populations. Malnourished children have many differences in their gastrointestinal anatomy and physiology compared to a healthy Western adult. These differences may affect the oral absorption of medicines and it is important to gather knowledge on these GI differences in order to develop biorelevant predictive methods for this vulnerable population.

A literature search was conducted within PubMed and Scopus to identify papers that describe how gastrointestinal physiology and anatomy is altered in malnourished children. Relevant data was extracted and a narrative review generated to describe how $\mathrm{Gl}$ differences may affect oral drug absorption.

Several differences in GI anatomy and physiology were reported in the literature including: reduced saliva secretion; increased gastric $\mathrm{pH}$; slower gastric emptying; increased levels of bacteria in the small intestine; reduced surface area of intestinal villi and increased intestinal permeability. Much of the data was more than 30 years old and referred to a heterogeneous malnourished population.

Sufficient data has been identified that will inform basic novel biorelevant methods to predict oral drug absorption in malnourished children. Further work is required to generate additional data to improve these models and also to verify the models with appropriate pharmacokinetic data.

\section{Keywords}

Malnourished, biopharmaceutics, children, absorption, gastrointestinal physiology 
When administering an oral drug product to a patient there are various factors which are important to ensure sufficient drug absorption; these include drug- and formulation-related parameters like drug solubility and permeability, drug product dissolution and gastrointestinal (GI) conditions including the composition and physicochemical properties of GI fluids, transit time and gut wall conditions relevant to metabolism, passive diffusion and active transport of drugs. GI physiology is therefore a key aspect that determines the in vivo performance of orally administered drugs.

Over the last decades, bio-predictive in vitro models have been established for predicting oral drug exposure for a number of compounds in adults after dosing either in fasted or fed conditions. There is a lack of such in vitro models for children, yet they are urgently required to better predict the in vivo performance of medicines in children to minimize the burden of clinical testing. When developing biorelevant in vitro media and models to simulate gastrointestinal conditions, the most appropriate environment needs to be represented. It is already known that there are differences in the gastrointestinal physiology of children in different age groups and in comparison to adults, which are accompanied by variable volumes, $\mathrm{pH}$ values and composition [1]; it is obvious that these differences will be different again in children suffering from diseases affecting $\mathrm{Gl}$ conditions or in malnourished children.

Malnutrition is a condition that is reported for several patients groups and can be quite common in hospitalized and elderly patients [2-4], pregnant and breastfeeding women, and children [5]. Due to their high nutritional requirements children are more susceptible to malnutrition than adults [6]. When designing appropriate bio-predictive in vitro models, it will be essential to consider the specific GI features of malnourished children. To date, a review of the GI conditions in malnourished children is not available. However, it is obvious, that malnutrition will alter the $\mathrm{Gl}$ environment. An altered $\mathrm{Gl}$ environment may affect pharmacokinetics of orally administered drugs, which in turn may affect efficacy and subsequent therapy. In basic terms the absorbable dose is influenced by the transit time; concentration of drug at the absorbing surface; surface area available for absorption and the permeability of the drug across the membrane. Differences in any of these parameters will affect the fraction of dose absorbed; hence predictions made in a healthy population may be very different to those where the GI parameters are different. A detailed understanding of the nature of specific $\mathrm{GI}$ parameters in malnourished children and of how much they may affect timing, site and extent of in vivo drug release seems to be one of the key factors for developing better oral medications for these children. Moreover, any in vitro and/or in silico models that consider these relevant GI parameters and would be applicable to predict the in vivo performance of orally administered drugs in malnourished children would be extremely beneficial with regard to increasing the safety and efficacy of oral medicines provided to this patient group.

The group of malnourished patients describes a heterogenous population. Malnutrition refers to deficiencies, excesses or imbalances in a person's intake of energy and/or nutrients. Malnutrition is divided into undernutriton, which includes stunting, wasting and underweight, and to overweight, which includes obesity and several diet relevant diseases as heart disease and cancer [7]. Undernutrition is caused by a lack of food in general, but also by inadequate, unhealthy diets and diseases including digestive and absorptive disorders resulting in 
micronutrient deficiencies. It makes children in particular much more vulnerable to disease and death and is responsible for approximately $50 \%$ of child mortality for those under five years old [8]. According to the latest United Nations International Children's Emergency Fund (UNICEF) / World Health Organisation (WHO) / World Bank Group Joint Child Malnutrition Estimates (May 2018) at least 202 million children worldwide are stunted or wasted [8].

In the literature, different criteria that have been applied to classify the nutritional status of children can be found (Table 1). According to current WHO criteria undernutrition can be present in 4 broad sub-forms, i.e. underweight (low weight-for-age), stunting (low height-forage), wasting (low weight-for-height) and deficiencies in vitamins and minerals [7].

The main hallmark of child malnutrition is growth retardation. Underweight, which represents low weight for age [7], is defined as a weight-for-age at least two standard deviations below the median weight based on the WHO child growth standards [9, 10]. "Stunting" is a term for chronic undernutrition and reflects low height for age [7]; defined as less than two standard deviations (SD) below the WHO standards [9, 10]. Acute undernutrition is termed "wasting", where moderate wasting is indicated by a weight between two and three SDs below the WHO standards [9, 10]. Severely wasted children have a weight of less than three SDs below the WHO standards or a mid-upper arm circumference (MUAC) of less than $115 \mathrm{~mm}$ in children of 6-60 months [9-11].

"Severe wasting" and bilateral oedema are independent diagnostic criteria for severe acute malnutrition (SAM), which is associated with a high risk of death and which requires urgent therapeutic feeding [11].

Protein-calorie malnutrition (PCM) is a specific subtype of SAM with a deficiency in macronutrients including protein, carbohydrates and fat. The WHO defines PCM, also known as protein energy malnutrition (PEM), as "a pathological condition that results from a lower ingestion of protein and calories, which occurs more frequently in children under five years of age". Severe PCM can be categorized into three principal clinical forms: (i) marasmus, an acute malnutrition characterized by severe wasting of fat and muscle and a gross underweight status; (ii) kwashiorkor presents with moderate growth retardation and bilateral pitting oedema; and (iii) marasmic kwashiorkor, the most severe form of PCM, a mixed form of both marasmus and kwashiorkor that is characterized by the presence of both wasting and bilateral pitting oedema [12].

PCM is a major public health problem affecting a high proportion of infants and older children world-wide and accounts for a high childhood morbidity and mortality in the developing countries. Its association with a wide spectrum of infections necessitates multiple drug therapies. Whereas the epidemiology of PCM has been extensively studied globally, the pathophysiological changes that may affect disposition of these drugs in malnourished children have not been reviewed in that much detail [13]. Oshikoya et al. [14] performed a systematic literature review to determine the effects of PCM on drug pharmacokinetics and concluded that there have been relatively few pharmacokinetic studies of drugs frequently used for treatment of children with PCM. However, they also present case examples in which decreased absorption of drugs such as carbamazepine [15], chloroquine [16], sulphadiazine [17], and chloramphenicol [18] have been reported in children with PCM when compared with healthy normal children and was attributed to morphological changes in the jejunum. In 
addition, treatment failure with artemether-lumefantrine was reported to be due to incomplete absorption in a malnourished child [19].

The aim of this review was to review the available data on the gastrointestinal anatomy and physiology of malnourished children relevant to the performance of orally administered

medicines. The focus was set on the screening of the particular features of undernutrition. Thus, in the following sections, the terms malnutrition and undernutrition can be regarded as interchangeable.

\section{TABLE 1 HERE PLEASE}

\section{Materials and methods}

A literature search was conducted within PubMed and Scopus using the search terms listed in Figure 1.

\section{FIGURE 1 HERE PLEASE}

Literature that provided original data on parameters that affect GI physiology associated with oral administration and absorption of drugs were included in further analysis. Specifically literature that reported aspects on gastrointestinal transport, motility or contents was sought. Studies where children had co-morbidities were also included in the analysis.

The search was conducted between September 2017 and March 2018. The search results were sorted by the authors (LF and EP-S) based on titles, abstracts or full text articles. Additional literature was obtained from reference lists of the identified articles or reviews on this topic.

For data extraction, studies were sorted according to whether they investigated oral cavity, gastric, small intestinal or large intestinal parameters. Further sorting permitted papers to be reviewed based on physiological parameters of these regions. Data was extracted from relevant papers to gather evidence that was summarized for each absorption parameter. There was too much heterogeneity in the identified literature which did not permit a systematic meta-analysis. Therefore the results could not be presented as a systematic review. Thus, a narrative review was selected to discuss reported effects of malnutrition that would impact on oral drug absorption considering the process from the initiation of oral ingestion to excretion. 


\section{Definition of malnutrition used in the papers identified.}

The wide range of criteria used to classify malnutrition was highlighted in Table 1 and the papers identified used a range of these criteria when reporting results. The NCHS criteria were the most commonly used with the Gomez index, the Wellcome classification and WHO child growth standards being used in multiple studies [23, 26-43].

\subsection{Oral cavity}

The oral cavity is the site of administration for oral medicines. Solid oral dosage forms are usually designed to be swallowed whole and to release the drug in the stomach and/or intestine. However, in children solid oral dosage forms might not be swallowed as rapidly as in adults. Thus, there is a chance that conditions in the oral cavity such as $\mathrm{pH}$, buffer capacity and composition of saliva affect both integrity and drug release from solid oral dosage forms and consequently affect in vivo drug release. Dosage form integrity could be affected by mechanical forces caused by tongue and palate as well as by the volume, $\mathrm{pH}$, buffer capacity and composition of saliva. Salivary $\mathrm{pH}$ and buffer capacity also play an important role in dental diseases and compared to healthy children a higher incidence of caries and other dental diseases have been observed in children with poverty driven malnutrition [44, 45]. Increased residence time and drug release in the oral cavity can increase systemic drug absorption across the oral mucosa. Therefore, mucosal integrity is also an important fact to consider when discussing drug administration to malnourished children.

\subsubsection{Salivary secretion}

Salivary secretion has been reported to be lower in malnourished compared to healthy children (Table 2a). Agarwal et al. reported that saliva collection took longer (30-40 minutes) following stimulation with citric acid and strawberry flavor in malnourished children compared to control children or children (aged 1-10 years) with milder forms of PEM (10 min) [30]. Other research found impaired saliva flow rates in severely malnourished children (aged 8-12 years and 1119 years) in both unstimulated and stimulated conditions [32, 35]. Saliva flow rates were reported to be linked with level of malnutrition and the reduction in flow rate was related to the severity of PEM [35]. However, it should be noted, that malnutrition is often linked with dehydration which independently of malnutrition is known to affect saliva secretion rate [ 46 , 47].

\subsubsection{Saliva pH and buffer capacity}

The $\mathrm{pH}$ of saliva was reported to be lower $(6.9 \pm 1.85)$ in underweight children compared to children of a normal weight (8.1 \pm 1.95$)$ (aged 6-12 years) [45] (Table 2b). In addition a reduction in salivary buffer capacity has been reported for this patient group [23, 32, 45].

\subsubsection{Saliva composition}

Concentrations of sodium, potassium, phosphate, hexosamines, fucose, sialic acid and total protein as well as amylase activity in malnourished children have been reported to be similar to those of a control group (aged 8-12 years) [23] (Table 2c). However, in the malnourished 
group (aged 8-12 years) significantly lower concentrations of calcium and chloride as well as a decreased amount of protein secreted per minute were found in paraffin-stimulated whole saliva [23]. Agarwal et al. detected a continuous fall of protein, ferritin and arginase activity in stimulated saliva that correlated with the severity of PEM [30].

Analyses of unstimulated whole saliva show contradictory results regarding the concentrations of total IgA: while Johansson et al. detected no differences in the concentrations of total IgA between the PEM group and the control group; McMurray et al. found reduced $\lg A$ concentrations in malnourished children (aged 1.5-2 years) [23, 31]. Salivary total protein, albumin and aminopeptidase were found in similar concentrations in all children by McMurray et al. and, Johansson et al. detected lower concentrations of anti-S. mutans IgA and lactoferrin as well as lower activity of the bacteria agglutinating protein (BAGP) in the PEM group.

\subsubsection{Oral mucosa integrity}

PEM appears to have multiple effects on the oral tissues and oral disease development [35]. Besides deficiencies in protein malnourished children PEM results in a lack of a number of essential vitamins and minerals that effect structures in the oral cavity. These include folate and other B complex vitamins; vitamins A, C, and D, calcium and fluoride. Consequently, besides impaired dentition, the nutritional status of the body is also associated with disturbances in other oral structures and presents with recurrent aphthous stomatitis, atrophic glossitis, painful, burning tongue characterized by inflammation and defoliation of the tongue, mucosal atrophy and oral ulcers $[35,48,49]$. There is very little data reported on the extent to which oral mucosa integrity is impaired in malnourished children. However, there is evidence that long-term chronic malnutrition causes a significant reduction in resistance and progressive damage to the oral mucosa that will result in reduced resistance to colonization and invasion of pathogenic microorganisms [50] and consequently will most probably also enhance permeability and therefore oromucosal drug absorption.

In summary, the imbalance between the supply of the nutrients and the body's demand results in several oral manifestations that could alter oral drug absorption. However, whereas the impacts on dentition are usually irreversible, in children rehabilitation will most likely result in complete recovery of oral mucosa integrity, saliva secretion and composition.

\section{TABLES 2 AND 3 HERE PLEASE}

\subsection{Stomach}

Volume, composition and physicochemical properties of gastric fluids are important factors regarding drug solubility and/or disintegration properties of solid oral dosage forms and thus can affect in vivo drug release. The motility pattern in the stomach and the secretion of gastric juices are directly dependent on food intake. Thus, it is necessary to differentiate between the fasted and fed state. Different nutrition habits or long periods of fasting lead to the assumption that gastric conditions in malnourished children may differ from conditions in healthy children, which may result in altered drug performance.

\subsubsection{Gastric contents}

Several studies have shown correlations between reduced fasted gastric acid secretion and malnutrition (Table 3a). Gracey et al. detected, that unstimulated gastric acid secretion was 
reduced in 9 of 14 malnourished children, while maximal acid output (60 minutes after pentagastrin stimulation) was below normal in all malnourished patients (aged 7-54 months) [42]. Gilman et al. reported that the fasted gastric acid output was lower in severely malnourished Bangladeshi children compared to better nourished children ( 0.22 vs $0.52 \mathrm{mEq}$ $\mathrm{HCl} / \mathrm{h}$ ) (aged $3.3 \pm 2.4$ years) [38]. In the children enrolled in the Gilman study, three weeks of nutritional rehabilitation lead to equivalent rates of fasted gastric acid secretion in the two groups showing that this is a reversible phenomenon [38]. At this point, it also should be noted that as for saliva secretion, impaired gastric secretion may relate to both malnutrition or dehydration of the children, which often cannot be clearly distinguished. Thus, the increase in gastric acid secretion could be also an effect of rehydration. Shashidhar et al. detected a decreased mean acid concentration in malnourished children (aged 12-60 months), here divided into kwashiorkor and marasmic children, under unstimulated and stimulated fasted conditions, although results show a high interindividual variability [51].

Under unstimulated fasted conditions the $\mathrm{pH}$ of gastric juice was above 4 in $76 \%$ of malnourished children; After nutritional rehabilitation this decreased to $69 \%$ in malnourished children compared to $55 \%$ in better-nourished children (aged $3.3 \pm 2.4$ years) [38]. Under stimulated conditions, the percentage of $\mathrm{pH}$ values above 4 was reduced: $26 \%$ in malnourished children, $24 \%$ in children after nutritional therapy and $0 \%$ in better-nourished children [38]. However, $\mathrm{pH}$ of gastric juice under stimulated fasted conditions is not equal to gastric $\mathrm{pH}$ under fed conditions. Thus, also the composition and quantity of food has an important impact on gastric $\mathrm{pH}$, especially when considering that there are various types of diets worldwide.

Peptic activity was reported to be significantly reduced in malnourished children under fasted conditions compared to well-nourished children (aged 12-60 months) [51] (Table 3a). Yet, shortly after stimulation of gastric secretion peptic activity rose in all children although it was still significantly decreased in malnourished children.

The reduced gastric output observed at baseline in severely malnourished children correlates to a higher incidence of infections [53] credited to the loss of the gastric acid barrier. The relationship between $H$. pylori infection and acidity within the stomach is complex; a low level of acidity in the stomach may increase the risk of infection in the gastrointestinal tract and also overgrowth of intestinal bacteria that may lead to diarrhoea. The prevalence of $H$. pylori in children is high in developing countries and since $H$. pylori infection is associated with an increased gastric $\mathrm{pH}$, it might be associated with malnutrition $[38,54,55]$.

In conclusion, available literature reports reduced gastric acid secretion in malnourished children, yet nutritional rehabilitation seems to result in an immediate improvement. It should be noted that nutritional rehabilitation typically results in rehydration of malnourished children. Malnourished children with watery diarrhea are usually treated with Rehydration Solution for Malnutrition (ReSoMal) [56]. Hence the increase in gastric acid secretion can be caused by both nutritional rehabilitation and rehydration. 


\subsubsection{Gastric emptying}

Investigations on gastric emptying in malnourished children show contradictory results (Table $3 b)$. Brunser et al. (within a review paper) stated without indicating a reference, that there are clinical observations showing that severe cases of malnutrition are characterized by gastric dilatation, slow emptying and vomiting [52]. Two studies investigating gastric emptying in marasmic, kwashiorkor and marasmic kwashiorkor children (aged 5-29 months and 7-45 months) showed that children with marasmus showed faster gastric emptying of a $5 \%(\mathrm{w} / \mathrm{v})$ glucose solution administered at a volume of $20 \mathrm{~mL} / \mathrm{kg}$ body weight compared to a control group. Severely malnourished children with kwashiorkor had no detectable abnormalities in gastric emptying, while in marasmic kwashiorkor children a tendency of delayed gastric emptying was observed [33, 34]. However, the power of these studies is limited, as the patient group was treated with different helminthics such as thiabendazole, which can evoke unspecific gastrointestinal disorders. In contrast, a significantly delayed gastric emptying in both marasmic and marasmic kwashiorkor children (aged $11.97 \pm 6.03$ months) was reported by Shaaban et al. [41]. In an ultrasonographic study they investigated the impact of nutritional rehabilitation on gastric emptying using a liquid (infant powdered milk formula) and a semisolid meal (milk, rice and high protein additive). They found a clear delay in gastric emptying of both liquid and semisolid food in marasmic and marasmic kwashiorkor children when compared to a control group (aged $11.97 \pm 6.03$ months). As could be expected, the delay in the gastric half emptying time was higher with the semisolid than with the liquid meals [41]. Nutritional rehabilitation was observed to speed up gastric emptying after $30 \pm 7$ days of nutritional treatment [41].

\subsection{Small intestine}

The small intestine is the site where maximal drug absorption occurs. Therefore, small intestinal conditions, such as luminal contents, surface area, transit time and mucosal permeability, can affect drug absorption and this information can help to develop predictive in vitro test designs as accurately as possible.

\subsubsection{Small intestinal contents}

The small intestinal contents influence the solubility of drugs which in turn will influence their absorption. Key components of small intestinal fluids are bile, enzymes and bacteria.

Mehta et al. analyzed the duodenal contents following a milk stimulus from sixty marasmic children between the ages of 9-42 months. Results showed significantly lower mean concentrations of conjugated bile acids $(1.36 \mathrm{mg} / \mathrm{mL}$ vs. $2.92 \mathrm{mg} / \mathrm{mL})$ and total bile acids in marasmic children compared to well-nourished children, while the concentration of free bile acids was increased $(0.60 \mathrm{mg} / \mathrm{mL}$ vs. $0.06 \mathrm{mg} / \mathrm{mL})$ (Table $4 \mathrm{a})$. Unfortunately, measured $\mathrm{pH}$ values weren't published, but the low pH of duodenal juice was mentioned [43]. Duodenal aspirates were analyzed from 18 severely protein calorie malnourished children (aged 15-64 months) to measure the contents with respect to the ability to achieve micellar solubilisation of lipids. The results showed that micellar lipid and fat absorption were low in PCM children due to lower levels of bile salts [57]. Children under 5 years of age with severe acute malnutrition were shown to have higher total serum bile acids compared to controls which relates to lower biliary secretion within the GI tract [58]. 
Sauniere et al. analyzed the duodenal contents of 25 children (1 month to 8 years) with acute symptoms of kwashiorkor. Bicarbonate and volume of duodenal juice of malnourished children were not different compared to the normal African population, while pancreatic enzymes, such as amylase and lipase, were significantly decreased, except for trypsin, which was not affected [59]. These results agree with the findings from 40 children (aged 9-51 months) with kwashiorkor where a wide variation in significant depression of enzyme activity was observed [60] (Table 4b).

Underproduction of acid in the stomach is the most likely cause of the bacterial overgrowth observed in the small intestine of undernourished children. The impact of high levels of bacteria in the small intestine include impaired nutrient absorption and risks of sub-efficacious oral vaccination in children aged 2 months to 5 years [61]. Several studies have reported higher than usual levels of viable bacteria within the small intestine in malnourished children (aged 9 months to 6 years) $[62,63]$ (Table 4c).

\subsubsection{Intestinal fluid output and dehydration in malnutrition}

The adult small intestine receives large quantities of fluid via dietary fluid intake or as secretions from salivary glands, stomach, pancreas, liver and the small intestine itself. The small intestinal epithelium absorbs about 6-7 $L$ of fluid per day. Only about $1.5 \mathrm{~L}$ enter the large intestine and are further reduced to less than $250 \mathrm{~mL} /$ day that are excreted with feces. If an individual has diarrhoea then osmoregulation is affected and the fluid within the intestine is not reabsorbed and the total intestinal fluid output is elevated to balance the osmolality to favour the absorption [64]. The small intestine is typically a site of net water absorption which has been observed in children with malnutrition. However, in cases of malnutrition that is associated with diarrhoea a net secretion of water was measured within the jejunum [65]. This alteration from net absorption to net secretion is likely to reduce the uptake of drugs within the $\mathrm{Gl}$ tract in cases of diarrhoea.

\section{TABLE 4 HERE PLEASE}

\subsubsection{Intestinal permeability}

Several studies done in the 1960-1970s showed that the shape of the small intestinal wall changed from regular long villi in healthy patients (villi are structures within the small intestine that increase the small intestinal surface area and, consequently, absorption) to irregular broader and shorter villi in malnourished children (0-3 years) and that the shape in malnutrition is associated with an overall smaller surface area compared to healthy tissue $[67,70,76]$ (Table 4e). However, increased permeation of cellular materials was seen suggesting that the intestine is more permeable in malnutrition $[67,70,76]$. As the severity of malnutrition increases so do the changes in the intestinal epithelium as the abnormalities in marasmus were mild in comparison to children (aged 0-3 years) with kwashiorkor [76]. Atrophy of villi in malnutrition has also been reported [68-70].

The Lactulose/Mannitol intestinal permeability test (L:M) is a commonly used technique to measure small intestinal function and can relate to changes in: a) small intestinal epithelial 
area; b) transcellular and paracellular transport and c) damage and permeability. Mannitol can be used as a marker to assess the mucosal absorptive area and lactulose to assess the integrity of the intestinal membrane. In some studies a lactulose/rhamnose test is used; rhamnose is similar to mannitol in that it is a monosaccharide and mucosal damage will reduce mannitol/rhamnose absorption whilst increasing permeability of lactulose.

A typical reference value for $L: M$ is 0.09 as this was defined as an upper normal limit in 30 healthy Dutch children (aged 0-16 years) [77]; values greater than 0.09 suggest enteropathy is present. Typically, lactulose (which is large) relates to the overall integrity of the membrane and mannitol can relate to the surface area or overall absorptive capacity of the membrane.

A study on 97 severely underweight Bangladeshi children (mean age 9 years) showed that the difference in mannitol absorption was greater than in lactulose absorption in malnourished children compared to healthy children; this relates to the loss of surface area which is consistent with the shape changes in the villi [27]. Intestinal biopsy studies [70, 78] in malnourished children show that kwashiorkor is associated with villous atrophy, decreased villous-crypt ratio and increased cellularity of the lamina propia; these changes impact the absorptive capacity as well as the overall surface area. Several studies have been conducted to investigate the intestinal permeability of malnourished children with or without diarrhea as well as before and after nutritional rehabilitation [26-28, 39, 40, 71, 79]. The damaged villi lead to a compromised intestinal wall that can be associated with absorption of large macromolecules that lead to immune- and inflammatory diseases. These diseases further damage the epithelial wall and reduce the barrier for absorption of drugs. Chronic intermittent diarrhea may be linked to carbohydrate malabsorption within the intestine due to the rapid intestinal transit times associated with diarrhea that may limit overall absorption in addition to the differences in the permeability across the membrane [76]. Brewster et al. showed that the combination of increased lactulose permeation and decreased L-rhamnose absorption results in a higher prevalence of diarrhea [71]. Behrens et al. found repeatedly increased intestinal permeability to disaccharides in a number of tests on 68 Gambian infants (aged 0-18 months), most of whom had at least one episode of diarrhea [40]. These findings lead to the conclusion that diarrhea is associated with mucosal damage, which results in compromised small intestinal barrier function and uptake of larger molecules, such as lactulose, through the site of damage [73]. Therefore, diarrhea seems to correlate with abnormally high L/R - ratios and thus with an increased intestinal permeability. Indeed, L/R - ratios of patients with diarrhea were observed to be above normal in several studies and correlated with the duration and frequency of diarrheic events and returned to normal with resolution of diarrhea [26, 40, 71]. It can be stated that malnutrition itself is associated with changes in the intestinal mucosa resulting in a reduced absorptive area and malabsorption, while diarrhea, which is often linked to malnutrition, is associated with mucosal damage.

Estimates of the prevalence of impaired intestinal permeability (higher values of L:M) range from $62-96 \%$ that results in poor growth among infants and young children in developing countries in sub-Saharan Africa and Asia (urinary L:M concentration ratio $>0.10-0.12$ ) [36, $74,80]$. Perturbed intestinal function is associated with the malabsorption of macro- and micronutrients, including fat, carbohydrates, and vitamins A, B12 and folate [81-83]. Wessells et al. (2013) study looked at zinc absorption in Burkinabe children (6-23 months) and also measured the lactulose/mannitol measures to assess intestinal integrity. The results showed that the absorption of zinc was linked to the L:M permeability test with a greater proportion of 
normal children showing higher levels of zinc absorption, however this was only significant different for the most severely affected children [29].

\subsubsection{Intestinal transit time}

The transit time through the small intestine is an important factor regarding the absorption of nutrients and drug products, as it affects the time that food and drugs have contact with the absorptive epithelium. In 1999 there was one study conducted regarding orocecal transit time in malnourished children. Myo-Khin et al. [37] used the lactulose breath hydrogen test to investigate orocecal transit in 90 Myanmar children between the ages of 1 and 5 years. After classification according to weight-for-age, length-for-age and weight-for-length indices, 31 children were defined to be malnourished, whose orocecal transit time was compared to transit time in well-nourished children. No significant difference between malnourished and wellnourished children was found. However, children with a history of diarrhoea were excluded from the study. As diarrhea and vomiting are two of the most common problems of PEM, as subsequent symptoms, a reduced retention of drugs and decrease of the transit time through the bowel can be expected [13].

\subsection{Colon}

No literature was identified that reported colonic transit time and colonic permeability in malnourished subjects. There are some studies suggesting that malnutrition is a risk factor for an increased incidence or duration of diarrhea [84-87]. As a consequence of diarrhea, colonic transit time in malnourished children might be shorter than in healthy children. However, some of the currently available reports on this topic are contradictive and the information available to date is not sufficient for a final conclusion. Another important point to consider in this discussion would be the alterations in the gut microbiome that are reported for severely malnourished children [88]. Future research should thus focus on expanding the knowledge in this field.

\section{Discussion}

Malnutrition can affect the gastrointestinal tract and subsequently, modify drug absorption. Therefore, malnutrition can have an indirect impact on drug toxicity or suboptimal therapeutic effects. Clearly, there is a pressing need to understand the impact of malnutrition in drug absorption.

The table below summarizes the findings from the literature and provides some implications for drug absorption in malnourished children.

\section{TABLE 5 HERE PLEASE}

The data identified within this search was generally quite old. Some general trends towards alterations in oral, gastric and small intestinal physiology were identified. Next to no 
information on the colon, another vital section of the GI tract, was available. There is very limited novel data available to really understand the implications of malnutrition on the absorption of orally administered medicines, yet this can be of great importance for such a vulnerable population. A systematic review on the impact of malnutrition on the pharmacokinetics of drugs used in children reported that the extent of absorption was increased in 8 drugs, yet this was attributed to be likely due to changes in clearance rather than the GI physiological changes [14]. However, these statements should be handled with care, since these 8 drugs had significantly different properties with regard to molecular weight, aqueous solubility and permeability in adults and represented candidates from all classes of the biopharmaceutics classification system (BCS). Consequently, at least for some of these APIs plasma levels in malnourished children are likely to be affected by GI physiology. Moreover, for several other compounds, examples where absorption in malnourished children was unchanged or reduced can be found in the literature [15-18].

Current in vitro tools that are used to predict oral drug absorption are based on adult physiologies and Western populations. The simulated gastrointestinal media and biorelevant dissolution methods available to date address $\mathrm{Gl}$ conditions in fasted and fed healthy European subjects [89-93]. Biorelevant in vitro tools addressing the particular GI features in malnourished children are currently unavailable. Therefore, there is a real need to develop biorelevant in vitro methods that reflect the conditions found in malnourished children and different types of malnutrition to better understand how drugs are absorbed in these critical and vulnerable populations. This will ensure the selection of adequate doses ensuring a safe and effective drug treatment of these children.

Biorelevant in silico tools using physiologically based pharmacokinetic modelling can provide a tool to better understand the disposition of drugs within a range of populations. There has been some work looking at simulating a malnourished population to explore drug pharmacokinetics [94]. In silico tools permit exploration of predicted pharmacokinetics when considering each different aspect of $\mathrm{Gl}$ physiology/anatomy for the population under investigation which can provide details on sensitivity to change based on the type of drug presented. Further work is required in this area.

\section{$5 \quad$ Conclusions}

Despite the significance of malnutrition as a global health issue this population has been neglected when considering efficacy of medicines used to treat malnourished children. Oral drug treatment during the acute phase of malnutrition needs to be carefully considered to ensure that the therapy is efficacious. Further work is required to develop biorelevant models that incorporate the findings from this review and to verify these in vitro and in silico models using appropriate pharmacokinetic data. All methods need to acknowledge the heterogeneity of malnutrition as a disease state as well as the age-related changes associated with paediatric populations. 


\section{Acknowledgements}

We would like to express our great appreciation to Colleen Emary and Dan Irvine from World Vision International for their valuable and constructive suggestions during the preparation of this manuscript. We further would like to show our gratitude to Albertina Arien, Sabine Inghelbrecht and Claire Mackie from Janssen Research and Development for their comments on an earlier version of this manuscript and for proofreading the paper.

\section{Financial support}

The research described in this paper was sponsored by a Ph.D. grant of Janssen Research \& Development, A Division of Janssen Pharmaceutica NV.

\section{References}

1. H.K. Batchelor, N. Fotaki, S. Klein, Paediatric oral biopharmaceutics: Key considerations and current challenges, Adv Drug Deliver Rev. 73 (2014) 102-126.

2. L.A. Barker, B.S. Gout, T.C. Crowe, Hospital malnutrition: prevalence, identification and impact on patients and the healthcare system, Int J Environ Res Public Health. 8 (2011) 514-27.

3. M. Hickson, Malnutrition and ageing, Postgrad Med J. 82 (2006) 2-8.

4. E. Agarwal, M. Miller, A. Yaxley, E. Isenring, Malnutrition in the elderly: a narrative review, Maturitas. 76 (2013) 296-302.

5. Y.H. Neggers, Trends in maternal mortality in the United States, Reprod Toxicol. 64 (2016) 72-6.

6. M. Blössner, M. de Onis, Malnutrition: quantifying the health impact at national and local levels, Geneva, WHO. 12 (2005).

7. www.who.int/features/qa/malnutrition/en/. (accessed 06 October 2017).

8. https://data.unicef.org/wp-content/uploads/2018/05/JME-2018-brochure-.pdf (accessed 25 May 2018).

9. J.I. Gordon, K.G. Dewey, D.A. Mills, R.M. Medzhitov, The human gut microbiota and undernutrition, Sci Transl Med. 4 (2012) 137ps12.

10. http://www.who.int/childgrowth/standards/en/. (accessed 25 May 2018).

11. http://apps.who.int/iris/bitstream/10665/44129/1/9789241598163_eng.pdf. (accessed 11 October 2017).

12. L. Rodriguez, E. Cervantes, R. Ortiz, Malnutrition and gastrointestinal and respiratory infections in children: a public health problem, Int J Environ Res Public Health. 8 (2011) 1174-205.

13. K.A. Oshikoya,I.O. Senbanjo, Pathophysiological changes that affect drug disposition in protein-energy malnourished children, Nutr Metab (Lond). 6 (2009) 50.

14. K.A. Oshikoya, H.M. Sammons, I. Choonara, A systematic review of pharmacokinetics studies in children with protein-energy malnutrition, Eur J Clin Pharmacol. 66 (2010) 1025-35. 
15. G. Bano, R.K. Raina, D.B. Sharma, Pharmacokinetics of carbamazepine in protein energy malnutrition, Pharmacology. 32 (1986) 232-6.

16. O. Walker, A.H. Dawodu, L.A. Salako, G. Alvan, A.O. Johnson, Single dose disposition of chloroquine in kwashiorkor and normal children--evidence for decreased absorption in kwashiorkor, Br J Clin Pharmacol. 23 (1987) 467-72.

17. S. Mehta, C.K. Nain, B. Sharma, V.S. Mathur, Metabolism of sulfadiazine in children with protein calorie malnutrition, Pharmacology. 21 (1980) 369-74.

18. M. Eriksson, L. Paalzow, P. Bolme, T.W. Mariam, Chloramphenicol pharmacokinetics in Ethiopian children of differing nutritional status, Eur J Clin Pharmacol. 24 (1983) 819-23.

19. N. Valecha, P. Srivastava, S.S. Mohanty, P. Mittra, S.K. Sharma, P.K. Tyagi, K. Pradhan, V. Dev, R. Singh, A.P. Dash, Y.D. Sharma, Therapeutic efficacy of artemether-lumefantrine in uncomplicated falciparum malaria in India, Malaria J. 8 (2009) 107-107.

20. F. Gomez, R. Ramos Galvan, S. Frenk, J. Cravioto Munoz, R. Chavez, J. Vazquez, Mortality in second and third degree malnutrition. 1956, Bull World Health Organ. 78 (2000) 1275-80.

21. D.S. McLaren, P.L. Pellett, W.W. Read, A simple scoring system for classifying the severe forms of protein-calorie malnutrition of early childhood, Lancet. 1 (1967) 5335.

22. J.C. Waterlow, Classification and definition of protein-energy malnutrition, Monogr Ser World Health Organ. (1976) 530-55.

23. I. Johansson, M. Lenander-Lumikari, A.K. Saellstrom, Saliva composition in Indian children with chronic protein-energy malnutrition, J Dent Res. 73 (1994) 11-9.

24. M.J. Dibley, J.B. Goldsby, N.W. Staehling, F.L. Trowbridge, Development of normalized curves for the international growth reference: historical and technical considerations, Am J Clin Nutr. 46 (1987) 736-48.

25. W.H.O.M.G.R.S. Group, WHO Child Growth Standards based on length/height, weight and age, Acta Paediatr Suppl. 450 (2006) 76-85.

26. R.T. Boaz, A.J. Joseph, G. Kang, A. Bose, Intestinal permeability in normally nourished and malnourished children with and without diarrhea, Indian Pediatr. 50 (2013) 152-3.

27. M.I. Hossain, B. Nahar, J.D. Hamadani, T. Ahmed, A.K. Roy, K.H. Brown, Intestinal mucosal permeability of severely underweight and nonmalnourished Bangladeshi children and effects of nutritional rehabilitation, J Pediatr Gastroenterol Nutr. 51 (2010) 638-44.

28. M.I. Hossain, R. Haque, D. Mondal, M. Mahfuz, A.S. Ahmed, M.M. Islam, R.L. Guerrant, W.A. Petri, Jr., T. Ahmed, Undernutrition, Vitamin A and Iron Deficiency Are Associated with Impaired Intestinal Mucosal Permeability in Young Bangladeshi Children Assessed by Lactulose/Mannitol Test, PLoS One. 11 (2016) e0164447.

29. K.R. Wessells, S.Y. Hess, N. Rouamba, Z.P. Ouedraogo, M. Kellogg, R. Goto, C. Duggan, J.B. Ouedraogo, K.H. Brown, Associations between intestinal mucosal function and changes in plasma zinc concentration following zinc supplementation, J Pediatr Gastroenterol Nutr. 57 (2013) 348-55.

30. P.K. Agarwal, K.N. Agarwal, D.K. Agarwal, Biochemical changes in saliva of malnourished children, Am J Clin Nutr. 39 (1984) 181-4. 
31. D.N. McMurray, H. Rey, L.J. Casazza, R.R. Watson, Effect of moderate malnutrition on concentrations of immunoglobulins and enzymes in tears and saliva of young Colombian children, Am J Clin Nutr. 30 (1977) 1944-8.

32. I. Johansson, A.K. Saelbtröm, B.P. Rajan, A. Parameswaran, Salivary Flow and Dental Caries in Indian Children Suffering from Chronic Malnutrition, Caries Res. 26 (1992) 38-43.

33. V.H. Franco, E.F. Collares, L.E. Troncon, Gastric emptying in children. III. Study on marasmic protein-calorie malnutrition, Arq Gastroenterol. 22 (1985) 88-92.

34. V.H. Franco, E.F. Collares, L.E. Troncon, Gastric emptying in children. IV. Studies on kwashiorkor and on marasmic kwashiorkor, Arq Gastroenterol. 23 (1986) 42-6.

35. W.J. Psoter, A.L. Spielman, B. Gebrian, R. St Jean, R.V. Katz, Effect of childhood malnutrition on salivary flow and pH, Arch Oral Biol. 53 (2008) 231-7.

36. R. Goto, C. Panter-Brick, C.A. Northrop-Clewes, R. Manahdhar, N.R. Tuladhar, Poor intestinal permeability in mildly stunted Nepali children: associations with weaning practices and Giardia lamblia infection, Br J Nutr. 88 (2002) 141-9.

37. Myo-Khin, T.D. Bolin, Tin-Oo, Thein-Win-Nyunt, S. Kyaw-Hla, Thein-Thein-Myint, Investigation of small-intestinal transit time in normal and malnourished children, $\mathrm{J}$ Gastroenterol. 34 (1999) 675-9.

38. R.H. Gilman, R. Partanen, K.H. Brown, W.M. Spira, S. Khanam, B. Greenberg, S.R. Bloom, A. Ali, Decreased gastric acid secretion and bacterial colonization of the stomach in severely malnourished Bangladeshi children, Gastroenterology. 94 (1988) 1308-14.

39. P.B. Sullivan, P.G. Lunn, C. Northrop-Clewes, P.T. Crowe, M.N. Marsh, G. Neale, Persistent diarrhea and malnutrition--the impact of treatment on small bowel structure and permeability, J Pediatr Gastroenterol Nutr. 14 (1992) 208-15.

40. R.H. Behrens, P.G. Lunn, C.A. Northrop, P.W. Hanlon, G. Neale, Factors affecting the integrity of the intestinal mucosa of Gambian children, Am J Clin Nutr. 45 (1987) 1433-41.

41. S.Y. Shaaban, M. Nassar, A. S Sawaby, H. El-Masry, A.F. Ghana, Ultrasonographic gastric emptying in protein energy malnutrition: Effect of type of meal and nutritional recovery. Vol. 58 (2004) 972-8.

42. M. Gracey, G.J. Cullity, Suharjono, Sunoto, The stomach in malnutrition, Arch Dis Child. 52 (1977) 325-7.

43. H.C. Mehta, A.S. Saini, H. Singh, P.S. Dhatt, Biochemical aspects of malabsorption in marasmus, Br J Nutr. 51 (1984) 1-6.

44. T.R. Ribeiro, K.S. Alves, A.C. de Miranda Mota, D.P. Costa, C.B. de Carvalho, C.F. Santos, A.J. Monteiro, C.S. Fonteles, Caries experience, mutans streptococci and total protein concentrations in children with protein-energy undernutrition, Aust Dent J. 59 (2014) 106-13.

45. A. Bud, E. Bud, D. Esian, S. Pop, A. Bechir, M. Pacurar, F. Curt-Mola, V. Tarmure, Interrelation between salivary $\mathrm{pH}$, buffer capacity and dental caries in underweight, normal weight and overweight children. Vol. 68 (2017) 1353-1356.

46. D. Fischer, J.A. Ship, The effect of dehydration on parotid salivary gland function, Spec Care Dentist. 17 (1997) 58-64.

47. N.P. Walsh, S.J. Laing, S.J. Oliver, J.C. Montague, R. Walters, J.L. Bilzon, Saliva parameters as potential indices of hydration status during acute dehydration, Med Sci Sports Exerc. 36 (2004) 1535-42. 
48. A. Sheetal, V.K. Hiremath, A.G. Patil, S. Sajjansetty, S.R. Kumar, Malnutrition and its oral outcome - a review, J Clin Diagn Res. 7 (2013) 178-80.

49. A.O. Ehizele, P.I. Ojehanon, O. Akhionbare, Nutrition and Oral Health, J Postgrad Med. 11 (2009) 76-82.

50. S.M. Słotwińska,R. Słotwiński, Host response, malnutrition and oral diseases. Part 2, Cent Eur J of Immunol. 39 (2014) 522-524.

51. S. Shashidhar, S. B Shah, P. T Acharya, Gastric acid, pH and pepsin in healthy and protein calorie malnourished children. Vol. 43 (1976) 145-51.

52. O. Brunser, M. Araya, J. Espinoza, Gastrointestinal Tract Changes in the Malnourished Child, Nestlé Nutr works se. 19 (1990) 261-276.

53. T.C. Martinsen, K. Bergh, H.L. Waldum, Gastric juice: a barrier against infectious diseases, Basic Clin Pharmacol Toxicol. 96 (2005) 94-102.

54. P.B. Sullivan, J.E. Thomas, D.G. Wight, G. Neale, E.J. Eastham, T. Corrah, N. LloydEvans, B.M. Greenwood, Helicobacter pylori in Gambian children with chronic diarrhoea and malnutrition, Arch Dis Child. 65 (1990) 189-91.

55. P. Sherman, S. Czinn, B. Drumm, F. Gottrand, E. Kawakami, A. Madrazo, G. Oderda, J.-K. Seo, P. Sullivan, S. Toyoda, L. Weaver, T.-C. Wu, Helicobacter pylori Infection in Children and Adolescents: Working Group Report of the First World Congress of Pediatric Gastroenterology, Hepatology, and Nutrition, J Pediatr Gastroenterol Nutr. 35 (2002) 128-133.

56. https://www.who.int/nutrition/publications/guide_inpatient_text.pdf. (accessed 17 January 2019).

57. R.E. Schneider,F.E. Viteri, Luminal events of lipid absorption in protein-calorie malnourished children; relationship with nutritional recovery and diarrhea. I. Capacity of the duodenal content to achieve micellar solubilization of lipids, Am J Clin Nutr. 27 (1974) $777-87$.

58. L. Zhang, W. Voskuijl, M. Mouzaki, A.K. Groen, J. Alexander, C. Bourdon, A. Wang, C.J. Versloot, V. Di Giovanni, R.J. Wanders, R. Bandsma, Impaired Bile Acid Homeostasis in Children with Severe Acute Malnutrition, PLoS One. 11 (2016) e0155143.

59. J.F. Sauniere, H. Sarles, Y. Attia, A. Lombardo, T.N. Yoman, R. Laugier, K. Manlan, J. Sahel, Exocrine pancreatic function of children from the Ivory Coast compared to French children. Effect of kwashiorkor, Dig Dis Sci. 31 (1986) 481-6.

60. M.D. Thompson,H.C. Trowell, Pancreatic enzyme activity in duodenal contents of children with a type of kwashiorkor, Lancet. 1 (1952) 1031-5.

61. R. Lagos, A. Fasano, S.S. Wasserman, V. Prado, O. San Martin, P. Abrego, G.A. Losonsky, S. Alegria, M.M. Levine, Effect of small bowel bacterial overgrowth on the immunogenicity of single-dose live oral cholera vaccine CVD 103-HgR, J Infect Dis. 180 (1999) 1709-12.

62. A.O. Redmond, J.D. Hansen, B. McHutchon, Abnormal bile salt metabolism in kwashiorkor, S Afr Med J. 46 (1972) 617-8.

63. B. Heyworth,J. Brown, Jejunal microflora in malnourished Gambian children, Arch Dis Child. 50 (1975) 27-33.

64. C.K. King, Glass, R., Bresee, J.S., Duggan, C., Managing Acute Gastroenteritis Among Children: Oral Dehydration, Maintenance, and Nutritional Therapy., CDC. 52(RR16) (2003) 1-16. 
65. W.P. James, B.S. Drasar, C. Miller, Physiological mechanism and pathogenesis of weanling diarrhea, Am J Clin Nutr. 25 (1972) 564-71.

66. I.U. Omoike,P.O. Abiodun, Upper small intestinal microflora in diarrhea and malnutrition in Nigerian children, J Pediatr Gastroenterol Nutr. 9 (1989) 314-21.

67. L.J. Mata, F. Jimenez, M. Cordon, R. Rosales, E. Prera, R.E. Schneider, F. Viteri, Gastrointestinal flora of children with protein--calorie malnutrition, Am J Clin Nutr. 25 (1972) 118-26.

68. D.I. Campbell, S.H. Murch, M. Elia, P.B. Sullivan, M.S. Sanyang, B. Jobarteh, P.G. Lunn, Chronic T cell-mediated enteropathy in rural west African children: relationship with nutritional status and small bowel function, Pediatr Res. 54 (2003) 306-11.

69. M. Farras, K. Chandwe, J. Mayneris-Perxachs, B. Amadi, J. Louis-Auguste, E. Besa, K. Zyambo, R. Guerrant, P. Kelly, J.R. Swann, Characterizing the metabolic phenotype of intestinal villus blunting in Zambian children with severe acute malnutrition and persistent diarrhea, PLoS One. 13 (2018) e0192092.

70. D. Burman, The jejunal mucosa in kwashiorkor, Arch Dis Child. 40 (1965) 526-31.

71. D.R. Brewster, M.J. Manary, I.S. Menzies, E.V. O'Loughlin, R.L. Henry, Intestinal permeability in kwashiorkor, Arch Dis Child. 76 (1997) 236-41.

72. K. Goto, F. Chew, B. Torun, J.M. Peerson, K.H. Brown, Epidemiology of altered intestinal permeability to lactulose and mannitol in Guatemalan infants, J Pediatr Gastroenterol Nutr. 28 (1999) 282-90.

73. P.G. Lunn, C.A. Northrop-Clewes, R.M. Downes, Recent developments in the nutritional management of diarrhoea. 2. Chronic diarrhoea and malnutrition in The Gambia: studies on intestinal permeability, Trans R Soc Trop Med Hyg. 85 (1991) 811.

74. M.J. Manary, S.A. Abrams, I.J. Griffin, M.M. Quimper, R.J. Shulman, M.G. Hamzo, Z. Chen, K. Maleta, M.J. Manary, Perturbed zinc homeostasis in rural 3-5-y-old Malawian children is associated with abnormalities in intestinal permeability attributed to tropical enteropathy, Pediatr Res. 67 (2010) 671-5.

75. K. Johansen, G. Stintzing, K.E. Magnusson, T. Sundqvist, F. Jalil, A. Murtaza, S.R. Khan, B.S. Lindblad, R. Mollby, E. Orusild, et al., Intestinal permeability assessed with polyethylene glycols in children with diarrhea due to rotavirus and common bacterial pathogens in a developing community, J Pediatr Gastroenterol Nutr. 9 (1989) 307-13.

76. J.V.M. Campos, U.F. Neto, F.R.S. Patricio, J. Wehba, A.A. Carvalho, M. Shiner, Jejunal mucosa in marasmic children. Clinical, pathological, and fine structural evaluation of the effect of protein-energy malnutrition and environmental contamination, Am J Clin Nutr. 32 (1979) 1575-1591.

77. R.M. van Elburg, J.J. Uil, F.T. Kokke, A.M. Mulder, W.G. van de Broek, C.J. Mulder, H.S. Heymans, Repeatability of the sugar-absorption test, using lactulose and mannitol, for measuring intestinal permeability for sugars, J Pediatr Gastroenterol Nutr. 20 (1995) 184-8.

78. J.P. Stanfield, M.S. Hutt, R. Tunnicliffe, Intestinal biopsy in kwashiorkor, Lancet. 2 (1965) 519-23.

79. D.R. Brewster, M.J. Manary, I.S. Menzies, R.L. Henry, E.V. O'Loughlin, Comparison of milk and maize based diets in kwashiorkor, Arch Dis Child. 76 (1997) 242-8.

80. P.G. Lunn, The impact of infection and nutrition on gut function and growth in childhood, Proc Nutr Soc. 59 (2000) 147-54. 
81. S.J. Baker, Subclinical intestinal malabsorption in developing countries, Bull World Health Organ. 54 (1976) 485-94.

82. K.H. Brown, L. Parry, M. Khatun, G. Ahmed, Lactose malabsorption in Bangladeshi village children: relation with age, history of recent diarrhea, nutritional status, and breast feeding, Am J Clin Nutr. 32 (1979) 1962-9.

83. A. Chacko, A. Begum, V.I. Mathan, Absorption of nutrient energy in southern Indian control subjects and patients with tropical sprue, Am J Clin Nutr. 40 (1984) 771-5.

84. J. Sepulveda, W. Willett, A. Munoz, Malnutrition and diarrhea. A longitudinal study among urban Mexican children, Am J Epidemiol. 127 (1988) 365-76.

85. D.L. Palmer, F.T. Koster, A.K. Alam, M.R. Islam, Nutritional status: a determinant of severity of diarrhea in patients with cholera, J Infect Dis. 134 (1976) 8-14.

86. S.C. Fuchs,C.G. Victora, Risk and prognostic factors for diarrheal disease in Brazilian infants: a special case-control design application, Cad Saude Publica. 18 (2002) 773-82.

87. R.L. Guerrant, J.B. Schorling, J.F. McAuliffe, M.A. de Souza, Diarrhea as a cause and an effect of malnutrition: diarrhea prevents catch-up growth and malnutrition increases diarrhea frequency and duration, Am J Trop Med Hyg. 47 (1992) 28-35.

88. S. Subramanian, S. Huq, T. Yatsunenko, R. Haque, M. Mahfuz, M.A. Alam, A. Benezra, J. DeStefano, M.F. Meier, B.D. Muegge, M.J. Barratt, L.G. VanArendonk, Q. Zhang, M.A. Province, W.A. Petri, Jr., T. Ahmed, J.I. Gordon, Persistent gut microbiota immaturity in malnourished Bangladeshi children, Nature. 510 (2014) 417-21.

89. M. Vertzoni, J. Dressman, J. Butler, J. Hempenstall, C. Reppas, Simulation of fasting gastric conditions and its importance for the in vivo dissolution of lipophilic compounds, Eur J Pharm Biopharm. 60 (2005) 413-7.

90. M. Vertzoni, A. Diakidou, M. Chatzilias, E. Soderlind, B. Abrahamsson, J.B.

Dressman, C. Reppas, Biorelevant media to simulate fluids in the ascending colon of humans and their usefulness in predicting intracolonic drug solubility, Pharm Res. 27 (2010) 2187-96.

91. J.B. Dressman, G.L. Amidon, C. Reppas, V.P. Shah, Dissolution testing as a prognostic tool for oral drug absorption: immediate release dosage forms, Pharm Res. 15 (1998) 11-22.

92. E. Jantratid, N. Janssen, C. Reppas, J.B. Dressman, Dissolution media simulating conditions in the proximal human gastrointestinal tract: an update, Pharm Res. 25 (2008) 1663-76.

93. S. Klein, The Use of Biorelevant Dissolution Media to Forecast the In Vivo Performance of a Drug, AAPS J. 12 (2010) 397-406.

94. N. Thuo, W. Ungphakorn, J. Karisa, S. Muchohi, A. Muturi, G. Kokwaro, A.H. Thomson, K. Maitland, Dosing regimens of oral ciprofloxacin for children with severe malnutrition: a population pharmacokinetic study with Monte Carlo simulation, J Antimicrob Chemother. 66 (2011) 2336-45. 


\section{Figure captions}

Figure 1: Search terms used in the literature search 